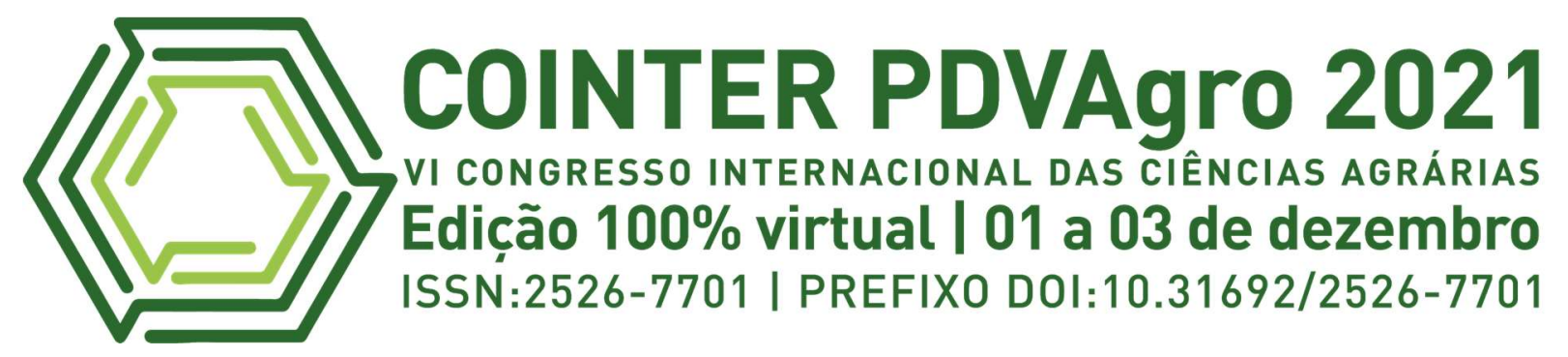

\title{
MODELAGEM MATEMÁTICA E CINÉTICA DE SECAGEM DE SEMENTES DE ABÓBORA
}

\author{
MODELADO MATEMÁTICO Y CINÉTICO DEL SECADO DE SEMILLAS DE \\ CALABAZA
}

\section{MATHEMATICAL AND KINETIC MODELING OF PUMPKIN SEEDS DRYING}

\author{
Apresentação: Comunicação Oral \\ Sâmela Leal Barros ${ }^{1}$; Maryana Melo Frota ${ }^{2}$; Mylena Olga Pessoa Melo ${ }^{3}$; Isabela Alves dos Santos ${ }^{4}$ Lucicleia \\ Barros de Vasconcelos; ${ }^{4}$ \\ DOI: https://doi.org/10.31692/2526-7701.VICOINTERPDVAgro.0146
}

\begin{abstract}
RESUMO
A abóbora possui uma importância socioeconômica que gira em torno do seu cultivo e, consequentemente, da sua comercialização. Durante o processamento, as sementes encontradas no interior do vegetal são descartadas, no entanto, são fontes de ácidos graxos e proteínas. Para a sua comercialização é necessário processos de secagem que preservem as qualidades físicas e nutricionais durante o armazenamento. Nessa perspectiva, o presente estudo teve o objetivo de ajustar diferentes modelos matemáticos aos valores experimentais da secagem de sementes de abóbora (Cucurbita máxima) e avaliar quais dos modelos melhor representa o fenômeno. A cinética de secagem das sementes foi realizada em estufa com circulação de ar, nas seguintes temperaturas: 50,60 e $70^{\circ} \mathrm{C}$. Aos dados experimentais foram ajustados três modelos empíricos, Page, Midilli e Henderson\&Pabis, avaliados quanto a magnitude do coeficiente de determinação $\left(\mathrm{R}^{2}\right)$ e do desvio quadrático médio (DQM) por meio do Software STATISTICA 12, utilizando a análise de regressão não-linear, pelo método QuasiNewton. O modelo de Midilli foi o que apresentou maiores valores de $\mathrm{R}^{2}$ e valores inferiores de $\mathrm{DQM}$ em comparação aos demais modelos, mostrando maior confiabilidade em representar o fenômeno de secagem de sementes de abóbora. $\mathrm{O}$ modelo de Henderson e Pabis apresentou uma distribuição tendenciosa de resíduos, indicando que não se adequa ao fenômeno estudado. Os tempos de secagem variaram de 450 a $600 \mathrm{~min}$. A maior temperatura estudada favoreceu a transferência de massa das sementes e reduziu o teor de umidade de equilíbrio dinâmico e o tempo de secagem. Percebeu-se a proporcionalidade dos parâmetros "a" e o " $\mathrm{k}$ " com a temperatura do ar de secagem, contudo, o parâmetro "b" sofreu diminuição com o acréscimo da temperatura. O parâmetro " $n$ ", não apresentou influência das temperaturas utilizadas.
\end{abstract}

Palavras-chave: Modelos matemáticos, Secagem convectiva, Sementes de abóbora.

\footnotetext{
${ }^{1}$ Doutoranda em Ciência e Tecnologia de Alimentos, Universidade Federal do Ceará, samelaleal@alu.ufc.br;

2 Engenharia de Alimentos, Universidade Federal do Ceará, mary.m.frota@gmail.com;

3 Doutoranda em Engenharia e Gestão dos Recursos Naturais, Universidade Federal de Campina Grande, mylenaopm@gmail.com;

${ }^{4}$ Doutoranda em Engenharia Química, Universidade Federal do Ceará, isabelaeq@gmail.com;

${ }^{5}$ Professora Dr ${ }^{\mathrm{a}}$, Universidade Federal do Ceará, lucicleia barros@ufc.br.
} 


\section{RESUMEN}

La calabaza tiene una importancia socioeconómica que gira en torno a su cultivo y, en consecuencia, a su comercialización. Durante el procesamiento, las semillas que se encuentran dentro de la planta se descartan, sin embargo, son fuente de ácidos grasos y proteínas. Para su comercialización son necesarios procesos de secado que conserven las cualidades físicas y nutricionales durante el almacenamiento. En esta perspectiva, el presente estudio tuvo como objetivo ajustar diferentes modelos matemáticos a los valores experimentales de secado de semillas de calabaza (Cucurbita maxima) y evaluar cuál de los modelos representa mejor el fenómeno. La cinética de secado de semillas se realizó en un invernadero con circulación de aire, a las siguientes temperaturas: 50,60 y $70{ }^{\circ} \mathrm{C}$. Se ajustaron tres modelos empíricos a los datos experimentales, Page, Midilli y Henderson y Pabis, evaluados para la magnitud del coeficiente de determinación (R2) y la desviación cuadrática media (DCM) utilizando el software STATISTICA 12, por medio de análisis de no regresión. lineal, por el método Quasi-Newton. El modelo Midilli mostró valores más altos de R2 y valores más bajos de DCM en comparación con los otros modelos, mostrando una mayor confiabilidad en la representación del fenómeno de secado de las semillas de calabaza. El modelo de Henderson y Pabis presentó una distribución de residuos sesgada, lo que indica que no se ajusta al fenómeno estudiado. Los tiempos de secado variaron de 450 a 600 min. La temperatura más alta estudiada favoreció la transferencia de masa de semillas y redujo el contenido de humedad de equilibrio dinámico y el tiempo de secado. Se notó la proporcionalidad de los parámetros "a" y "k" con la temperatura del aire de secado, sin embargo, el parámetro "b" disminuyó con el aumento de temperatura. El parámetro "n" no mostró influencia de las temperaturas utilizadas.

Palabras clave: Modelos matemáticos, Secado convectivo, Semillas de calabaza.

\section{ABSTRACT}

The squash has a socioeconomic importance that revolves around its cultivation and, consequently, its commercialization. During processing, the seeds found inside the plant are discarded, however, they are sources of fatty acids and proteins. For its commercialization, drying processes that preserve the physical and nutritional qualities during storage are necessary. In this perspective, the present study aimed to adjust different mathematical models to the experimental values of drying pumpkin seeds (Cucurbita maxima) and to evaluate which of the models best represents the phenomenon. Seed drying kinetics was performed in a greenhouse with air circulation, at the following temperatures: 50,60 and $70^{\circ} \mathrm{C}$. Three empirical models were fitted to the experimental data, Page, Midilli and Henderson and Pabis, evaluated for the magnitude of the coefficient of determination (R2) and the mean square deviation (MQD) using the STATISTICA 12 Software, using non-regression analysis. linear, by the Quasi-Newton method. The Midilli model showed higher values of R2 and lower values of MQD compared to the other models, showing greater reliability in representing the drying phenomenon of pumpkin seeds. The Henderson and Pabis model presented a biased distribution of residues, indicating that it does not fit the studied phenomenon. Drying times ranged from 450 to $600 \mathrm{~min}$. The highest temperature studied favored seed mass transfer and reduced the dynamic equilibrium moisture content and drying time. It was noticed the proportionality of parameters "a" and " $\mathrm{k}$ " with the temperature of the drying air, however, parameter "b" decreased with the increase in temperature. The parameter " $n$ " did not show influence of the temperatures used.

Keywords: Mathematical models, Convective drying, Pumpkin seeds.

\section{INTRODUÇÃO}

A abóbora (Cucurbita maxima) é um fruto que pertence à família Cucurbitaceae. É rica em polissacarídeos, vitaminas, sais minerais, pectina e carbonato (VALE et. al., 2019). Seu cultivo em grande escala predomina no Nordeste brasileiro, onde são muito consumidas na dieta da população quando maduras (MALDONADE et al., 2019). Portanto, a importância socioeconômica em torno do cultivo das espécies de abóboras é enorme, uma vez que esse 
vegetal está entre os dez mais cultivados (VALE et. al., 2019).

$\mathrm{Na}$ comercialização dos frutos de abóbora, as sementes pertencentes são subprodutos do processamento com alta qualidade, com propriedades funcionais e tecnológicas de interesse. Entretanto, geralmente são descartadas (VIEIRA et. al., 2019). São consideradas fontes ricas em ácidos graxos e proteínas, possuindo alto teor de aminoácidos essenciais (HERNÁNDEZSANTOS et. al., 2016), fibras e compostos antioxidantes (AMARAL et al., 2019). Para Silva et. al. (2020), a comercialização das sementes de abóbora requer conhecimentos sobre seu armazenamento e o processo de secagem para a preservação da qualidade e dos componentes de interesse.

A secagem é uma operação unitária de grande importância na indústria de sementes e subprodutos. Consiste na evaporação da água do material, com a finalidade de preservar a qualidade e estabilidade do produto (SILVA et. al., 2020). Dessa forma, a secagem convectiva se torna atrativa, sendo amplamente utilizada por apresentar inúmeras vantagens, basicamente por ser um método de baixo custo, simples e eficiente para a conservação dos produtos (SILVA, 2016).

O controle de sistemas de secagem é de fundamental importância para o desenvolvimento e melhorias de equipamentos e efetividade da aplicação comercial (ALVES, 2019). Para isso, são realizadas cinéticas de secagem para a obtenção de informações que apresentem o comportamento do material seco, representado por curvas e taxas de secagem. Através da avaliação do ajuste dos modelos matemáticos aos dados experimentais de secagem é possível identificar qual modelo apresenta maior confiabilidade na descrição do processo, podendo ser utilizado posteriormente para simular o processo de secagem para o produto, com relação ao tempo requerido para que o produto alcance determinado teor de umidade nas condições avaliadas, podendo reduzir o tempo de residência do produto na estufa. Neste sentido, pode-se inferir também que as cinéticas de secagem possibilitam a otimização do processo e melhoria na qualidade do produto final. A literatura disponibiliza diversos modelos matemáticos que descrevem o processo de perda de umidade por secagem de produtos agrícolas (SILVA, 2016).

Nesse sentido, o objetivo do presente trabalho consistiu em realizar a cinética de secagem das sementes de abóbora em diferentes temperaturas, aplicando os dados experimentais obtidos em modelos matemáticos empíricos, considerando a influência da secagem em suas dimensões.

\section{FUNDAMENTAÇÃO TEÓRICA}




\section{Abóbora (Cucurbita máxima)}

A abóbora é considerada uma das hortaliças mais vendidas e consumidas, isso se deve a facilidade no manuseio e produção, cultivo em todas as épocas do ano e sua longa durabilidade após a colheita, podendo ser armazenada em temperatura ambiente (ALVES, 2019).

É um vegetal que pode apresentar diversas aplicações de consumo, in natura, cozida ou como matéria-prima em produtos como: purês, geléias, bolos, chips e doces. Além de ter um papel importante na alimentação de muitos países, a abóbora possui valor socioeconômico, uma vez que seu cultivo o ano inteiro gera empregos de mão de obra para o seu plantio, se estendendo até a comercialização (RESENDE, BORGES e GONÇALVES 2013).

Devido a sua composição abranger uma variedade de nutrientes importantes, um dos apelos relacionados ao consumo de abóbora é com relação a sua funcionalidade nos organismos. Além disso, as sementes da abóbora têm se destacado nutricionalmente, mas também na sua importância funcional e tecnológica. Possuem alto teor de lipídios, aminoácidos, fibras e compostos antioxidantes (AMARAL et al., 2019). Nesses casos, o processo de secagem é especialmente importante para a preservação e transformação do material (BENSEDDIK et al., 2018).

\section{Secagem de produto agrícola e modelagem matemática}

Os produtos de origem vegetal, apresentam majoritariamente um alto teor de umidade e de atividade de água, fatores que propiciam o crescimento de fungos, bactérias e a ocorrência de reações químicas degradativas. Para minimizar a ocorrência destes problemas durante o armazenamento, efetua-se a remoção parcial de água no produto. Este processo é denominado por secagem, que é um dos métodos mais utilizados para promover o aumento da vida útil de produtos de origem vegetal, além de ser o mais antigo. O processo de secagem é aplicado a produtos agrícolas frescos, como frutas, vegetais e folhas, que contêm principalmente uma alta porcentagem de água ligada e não ligada (BABU et al., 2018).

Os alimentos possuem comportamentos distintos quando submetidos à secagem. As propriedades físicas e químicas, a geometria, a espessura e a composição determinam todo o processo. Dessa forma, modelos matemáticos são aplicados para fornecer informações precisas que representam a perda de umidade do produto durante o período de secagem (SILVA, 2016).

\section{METODOLOGIA}

\section{Matéria-prima}

As sementes de abóbora (Cucurbita máxima) foram fornecidas por produtor local, 
anteriormente consideradas como resíduos oriundos do processamento mínimo de alimentos de origem vegetal.

\section{Local de realização}

As sementes foram conduzidas ao laboratório de frutos tropicais (pertencente a Unidade Acadêmica de Engenharia de Alimentos, na Universidade Federal do Ceará), onde foram lavadas em água corrente e sanitizadas em solução de hipoclorito de sódio a 200 ppm por 15 minutos para remoção de sujidades.

A obtenção das farinhas de sementes de abóbora foi realizada por meio das etapas apresentadas no fluxograma da Figura 01.

Figura 01: Fluxograma de etapas para obtenção das farinhas de semente de abóbora.

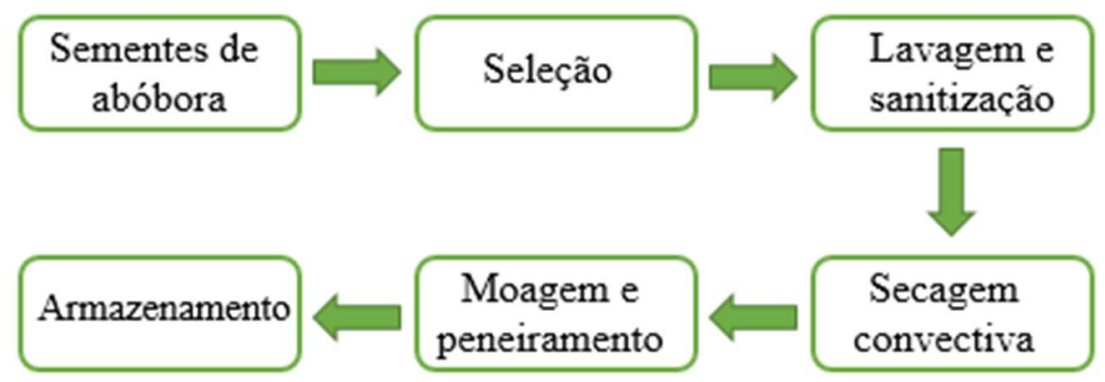

Fonte: Própria (2019).

\section{Processo de secagem convectiva}

Os ensaios de secagem foram realizados em triplicata, em estufa com circulação de ar, nas seguintes temperaturas: 50,60 e $70^{\circ} \mathrm{C}$. As sementes de abóbora foram pesadas em uma bandeja com o material distribuído de forma que permitisse que a secagem ocorresse da maneira mais uniforme possível, como observado na Figura 02.

Figura 02: Sementes de abóbora distribuidas em placa de Petri

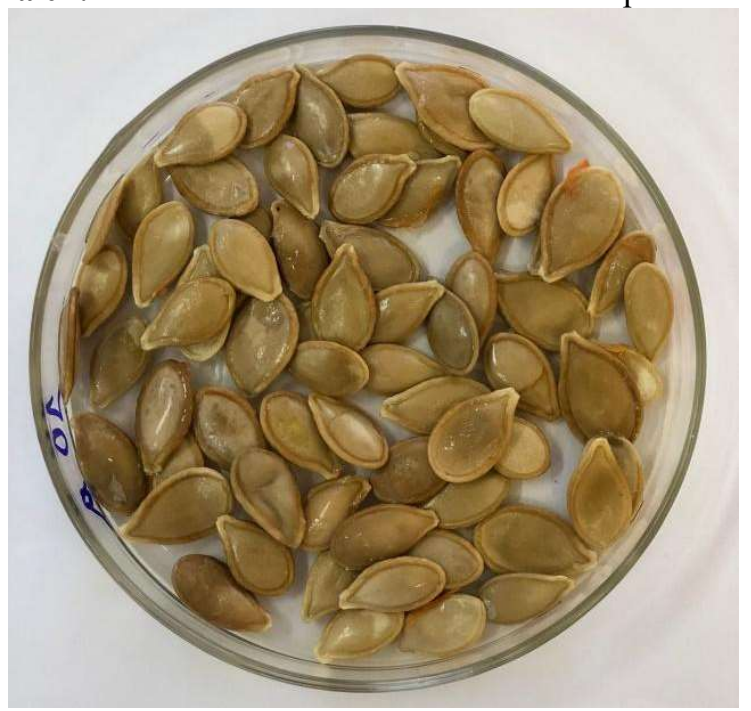


Fonte: Própria (2021)

O conjunto (placa de Petri + amostra) foi pesado até que não existisse mais variação significativa da massa da amostra em intervalos de tempo de 5 minutos (por meia hora), 10 minutos (por meia hora), 30 minutos (por 1 hora e meia) e 1 hora até atingir o teor de umidade de equilíbrio. Os dados experimentais foram expressos na forma de razão de teor de água ( $\mathrm{R} X)$ conforme mostra a Equação (1):

$$
R X=\frac{X_{t}-X_{e}}{X_{0}-X_{e}}
$$

Em que: $\mathrm{Xt}=$ teor de água no instante $\mathrm{t}$, base seca, bs; $\mathrm{Xe}=$ teor de água de equilíbrio, bs; Xo=teor de água inicial, bs.

A Tabela 01 apresenta as funções empíricas $f(\mathrm{t}, \mathrm{a}, \mathrm{b})$ que foram ajustadas aos conjuntos de dados experimentais através de regressão não linear.

Tabela 01: Modelos matemáticos utilizados na descrição da cinética de secagem

\begin{tabular}{|c|c|c|c|}
\hline Modelo & Equações empíricas & Referência & \\
\hline Page & $\mathrm{RX}=\exp \left(-\mathrm{k} \cdot \mathrm{t}^{\mathrm{n}}\right)$ & Page (1949) & (Eq. 2) \\
\hline Midilli & $\mathrm{RX}=\mathrm{a} \cdot \exp \left(-\mathrm{k} \cdot \mathrm{t}^{\mathrm{n}}\right)+\mathrm{b} \cdot \mathrm{t}$ & Midilli et al. (2002) & (Eq. 3) \\
\hline Henderson e Pabis & $\mathrm{RX}=\mathrm{a} . \exp (-\mathrm{k} . \mathrm{t})$ & Henderson e Pabis (1961) & (Eq. 4) \\
\hline
\end{tabular}

Para análise dos ajustes dos modelos matemáticos aos dados experimentais, foi utilizado o programa computacional STATISTICA, versão 12, utilizando-se a análise de regressão nãolinear, pelo método Quasi-Newton. Os modelos foram selecionados tomando-se como parâmetro a magnitude do coeficiente de determinação $\left(\mathrm{R}^{2}\right)$ e do desvio quadrático médio (DQM) (Equação 5).

$$
D Q M=\sqrt{\sum_{i=1}^{n} \frac{\left(R X_{\exp }-R X_{\text {pred }}\right)^{2}}{N}}
$$

Em que: $\mathrm{RX}_{\exp }=$ Razão de teor de água obtida experimentalmente; $\mathrm{RX}_{\text {pre }}=$ Razão de teor de água predita pelo modelo matemático; $\mathrm{N}$ = número de observações ao longo da cinética de secagem. 


\section{Taxa de secagem}

A partir do cálculo da derivada do modelo de Page, expresso na Tabela 01, foi obtida a equação 6, que representa as taxas de secagem do produto durante o tempo do processo.

$$
d R X / d t=-K \cdot n \cdot t^{n-1} e^{-k t^{n}}
$$

\section{Moagem e padronização da granulometria}

Para a realização das análises físico-químicas, as sementes foram trituradas em moinho analítico IKA A11 ${ }^{\circledR}$ basic mill após o processo de secagem e peneiradas em malha $18(1,0 \mathrm{~mm})$ para a obtenção de um pó de granulometria homogênea.

\section{RESULTADOS E DISCUSSÃO}

Através da Tabela 02 foram expressos os valores obtidos com relação ao ajuste dos modelos matemáticos empíricos aos conjuntos de dados experimentais, das cinéticas de secagem convectiva de sementes de abóbora, utilizando diferentes temperaturas do ar de secagem $\left(50,60\right.$ e $\left.70{ }^{\circ} \mathrm{C}\right)$.

Tabela 02: Parâmetros de ajuste dos modelos matemáticos utilizados na predição da cinética de secagem das

\begin{tabular}{cccccc}
\multicolumn{5}{c}{ sementes de abóbora nas temperaturas de 50,60,70 ${ }^{\circ} \mathrm{C}}$. \\
\hline Modelo & Temperatura & \multicolumn{4}{c}{ Parâmetro } \\
& & $\mathrm{a}$ & $\mathrm{b}$ & $\mathrm{k}$ & $\mathrm{n}$ \\
\multirow{2}{*}{ Page } & $50^{\circ} \mathrm{C}$ & - & - & 0,0127 & 1,0101 \\
& $60{ }^{\circ} \mathrm{C}$ & - & - & 0,0200 & 1,0213 \\
& $70^{\circ} \mathrm{C}$ & - & - & 0,0297 & 0,9712 \\
\multirow{2}{*}{ Henderson \& Pabis } & $50^{\circ} \mathrm{C}$ & 0,9931 & - & 0,0133 & - \\
& $60{ }^{\circ} \mathrm{C}$ & 0,9948 & - & 0,0215 & - \\
\multirow{2}{*}{ Midilli } & $70^{\circ} \mathrm{C}$ & 1,0050 & - & 0,0266 & - \\
& $50^{\circ} \mathrm{C}$ & 0,9753 & 0,0001 & 0,0123 & 1,0198 \\
& $60^{\circ} \mathrm{C}$ & 1,0021 & 0,0000 & 0,0161 & 1,0718 \\
& $70^{\circ} \mathrm{C}$ & 1,0031 & 0,0000 & 0,0299 & 0,9707 \\
\hline
\end{tabular}

Fonte: Própria (2021)

Na Tabela 02, observa-se expresso o parâmetro "a" nos modelos de Henderson\&Pabis e Midilii, com relação a este parâmetro verifica-se que ocorre uma tendência ao aumento devido ao acréscimo da temperatura aplicada ao ar de secagem, que variou de 0,97 (na temperatura de $50{ }^{\circ} \mathrm{C}$ ) a 1,00 (na temperatura de $70{ }^{\circ} \mathrm{C}$ ) no modelo de Midilli e de 0,99 (na temperatura de 50 ${ }^{\circ} \mathrm{C}$ ) a 1,00 (na temperatura de $70{ }^{\circ} \mathrm{C}$ ) no modelo de Henderson\&Pabis. Comportamento semelhante foi observado por Silva et al. (2019), ao efetuarem o processo de secagem em 
cascas e albedo de maracujá, utilizando as temperaturas de 70 e $80^{\circ} \mathrm{C}$.

De acordo com Santos et al. (2019), o parâmetro "a" é fortemente influenciado pela temperatura do ar de secagem, pois este parâmetro representa a taxa de secagem nos modelos matemáticos empregados. $\mathrm{O}$ aumento no valor do parâmetro pode indicar uma diminuição do tempo requerido para que as amostras alcancem o teor de umidade de equilíbrio, obtido quando ocorre um RX próximo de 0 . Sendo assim, quanto maior for o "a" menor será o tempo de secagem do produto.

Verifica-se através da Tabela 02, que o parâmetro $K$ apresenta uma tendência de aumento com relação ao aumento de temperatura do ar de secagem em todos os modelos matemáticos aplicados. Portanto, as amostras que foram submetidas a temperatura de $70{ }^{\circ} \mathrm{C}$ apresentaram valores superiores com relação a este parâmetro, que variaram sutilmente de acordo com o modelo matemático $(0,0215$ a 0,0299). As amostras submetidas a temperatura de $50{ }^{\circ} \mathrm{C}$ apresentaram valores inferiores de $\mathrm{K}$, que variaram de 0,0123 a 0,0133 . Comportamento semelhante foi observado por Moscon et al. (2017), ao realizarem a cinética de secagem de grãos de quinoa nas temperaturas de $40,50,60$ e $70^{\circ} \mathrm{C}$.

O coeficiente " $n$ " variou ligeiramente entre 0,97 a 1,01, contudo não foi verificada correlação entre os valores deste coeficiente e as temperaturas do ar de secagem. De acordo com Santos et al. (2020), o coeficiente " $n$ " representa o gradiente existente entre o pressões de vapor do ar e pressão de vapor do material, teoricamente o aumento de temperatura do ar de secagem deveria causar um acréscimo neste coeficiente, levando a uma maior taxa de remoção de água do produto. Apesar disto, os autores não verificaram influência da temperatura nos valores obtidos para o parâmetro $\mathrm{n}$ durante o processo de secagem convectiva de arroz vermelho.

Com relação ao parâmetro "b", obteve-se valores que variaram de 0 a 0,001. Em que o maior valor foi observado na temperatura de $50{ }^{\circ} \mathrm{C}$ do ar de secagem, decrescendo com o aumento da temperatura. De acordo com Moreira et al. (2018), o parâmetro "b" é uma constante de proporcionalidade entre a taxa de secagem e a razão de umidade.

A Tabela 03 expressa os parâmetros estatísticos obtidos a partir dos modelos matemáticos de Page, Henderson\&Pabis e Midilli aos dados experimentais da secagem de sementes de abóbora nas temperaturas de 50,60 e $70^{\circ} \mathrm{C}$.

Tabela 03: Parâmetros estatísticos dos modelos matemáticos utilizados na predição da cinética de secagem das 
sementes de abóbora nas temperaturas de 50,60 e $70{ }^{\circ} \mathrm{C}$

\begin{tabular}{cccr}
\hline Modelo & Temperatura & \multicolumn{2}{c}{ Parâmetro } \\
& & $\mathrm{R}^{2}$ & $\mathrm{DQM}$ \\
Page & $50^{\circ} \mathrm{C}$ & 0,9991 & 0,0271 \\
& $60^{\circ} \mathrm{C}$ & 0,9981 & 0,0024 \\
& $70^{\circ} \mathrm{C}$ & 0,9987 & 0,0105 \\
Henderson \& Pabis & $50^{\circ} \mathrm{C}$ & 0,9991 & 0,0246 \\
& $60^{\circ} \mathrm{C}$ & 0,9981 & 0,0007 \\
Midilli & $70^{\circ} \mathrm{C}$ & 0,9986 & 0,0172 \\
& $50^{\circ} \mathrm{C}$ & 0,9993 & 0,0052 \\
& $60^{\circ} \mathrm{C}$ & 0,9985 & 0,0006 \\
& $70^{\circ} \mathrm{C}$ & 0,9988 & 0,0023 \\
\hline
\end{tabular}

Fonte: Própria (2021)

Através da observação dos parâmetros estatísticos, pode-se observar que todos os modelos matemáticos apresentaram ajuste satisfatório aos dados experimentais. Pois, os valores apresentados para os coeficientes de determinação $\left(R^{2}\right)$ dos modelos foram superiores a 0,99 e os valores do desvio quadrático médio (DQM) foram inferiores a 0,02. Ao comparar os resultados obtidos através do ajuste dos modelos matemáticos empíricos para os parâmetros estatísticos, pode-se inferir que o modelo de Midilli apresentou valores superiores de $\mathrm{R}^{2}$, que variou de 0,9985 a 0,9993. Com relação ao DQM, o modelo de Midilli apresentou valores inferiores quando comparado aos demais modelos matemáticos, indicando que o modelo apresenta maior confiabilidade na descrição do processo de secagem de sementes de abóbora.

Segundo Alves et al. (2019), quanto maior for o coeficiente de determinação $\left(\mathrm{R}^{2}\right)$, maior a confiabilidade do modelo matemático na descrição do processo de secagem. Ainda pode-se afirmar que valores de $\mathrm{R}^{2}$ superiores 0,95 indicam que o modelo pode ser utilizado para prever com precisão o comportamento do produto com relação a perda de umidade durante o processo de secagem. Porém, este parâmetro deve ser avaliado em conjunto com algum parâmetro estatístico como o qui-quadrado $\left(\chi^{2}\right)$ ou desvio quadrado médio (DQM), em que valores de $\mathrm{R}^{2}$ próximos a 1 e valores de DQM próximos a 0 indicam maior eficiência do modelo matemático na descrição do processo de secagem convectiva.

Tabela 04: Distribuição de resíduos das equações dos modelos matemáticos utilizados na predição da cinética de secagem das sementes de abóbora nas temperaturas de 50,60 e $70{ }^{\circ} \mathrm{C}$.

\section{Modelos}

$\mathrm{T}\left({ }^{\circ} \mathrm{C}\right) \quad$ Midili $\quad$ Page Henderson\&Pabis




\begin{tabular}{cccc}
50 & Aleatória & Aleatória & Aleatória \\
60 & Aleatória & Aleatória & Aleatória \\
70 & Aleatória & Aleatória & Tendenciosa \\
\hline
\end{tabular}

Fonte: Própria (2021)

Através da Tabela 04, verifica-se que os modelos matemáticos de Midilli e Page apresentam distribuição aleatória de resíduos para todas as temperaturas aplicadas. Porém, o modelo de Henderson e Pabis apresentou distribuição tendenciosa.

Quando um modelo matemático apresenta distribuição de resíduos aleatória, pode ser considerado satisfatório para representar o fenômeno estudado, pois indica que os valores dos resíduos são representados próximos da faixa horizontal (em torno de zero) e, caso o modelo apresente distribuição dos resíduos de forma tendenciosa, o mesmo não é adequado para representar o fenômeno em questão (GONELI, 2008; MARTINS et al., 2015).

A Figura 03 apresenta a curva de secagem das sementes de abóbora para a temperatura de $50^{\circ} \mathrm{C}$.

Figura 03: Curva de secagem das sementes de abóbora obtida pelo modelo matemático de Midilli na temperatura de $50{ }^{\circ} \mathrm{C}$

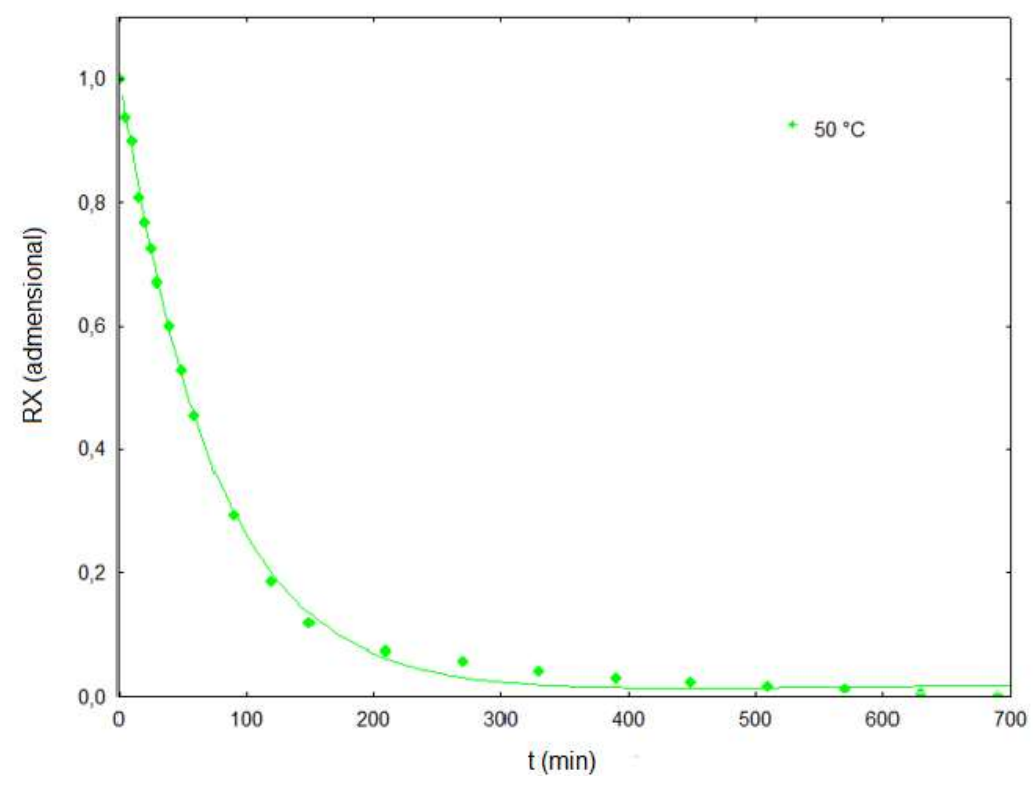

Fonte: Própria (2021)

Ao observar a Figura 03, pode-se verificar que o teor de umidade de equilíbrio foi obtido após 600 minutos de secagem na temperatura de $50{ }^{\circ} \mathrm{C}$.

A Figura 04 apresenta a curva de secagem das sementes de abóbora para a temperatura de $60^{\circ} \mathrm{C}$.

Figura 04: Curva de secagem das sementes de abóbora obtida pelo modelo matemático de Midilli na 


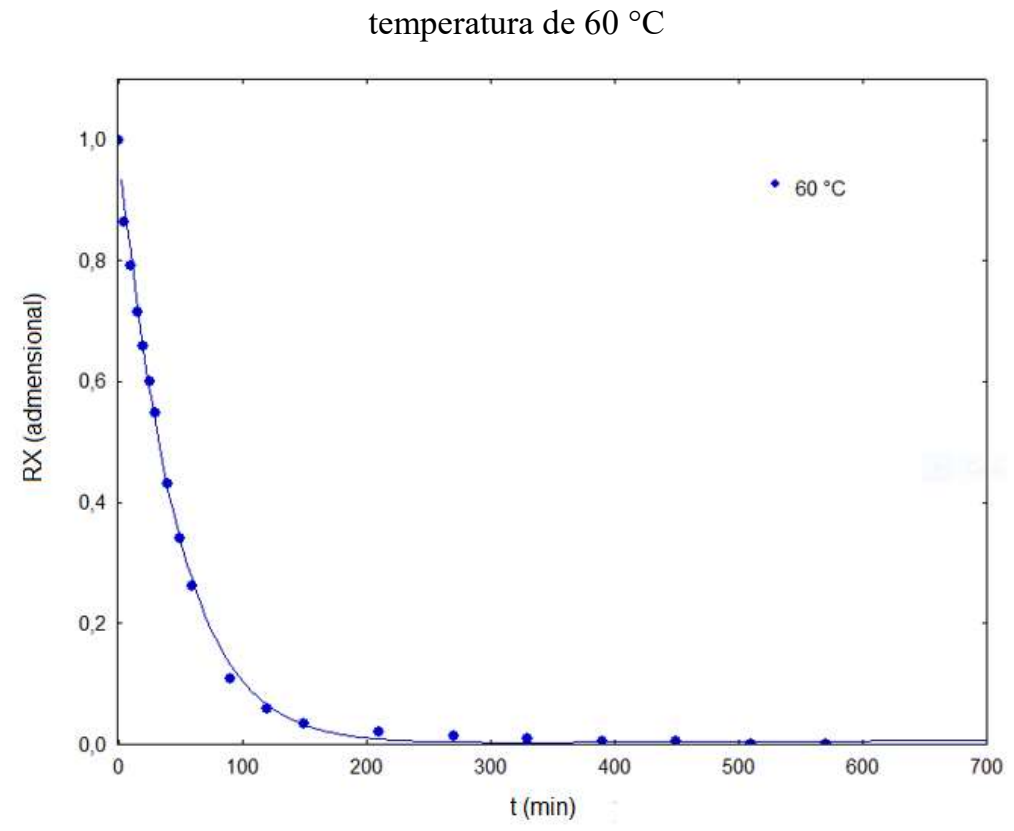

Fonte: Própria (2021)

Ao observar a Figura 04, pode-se verificar que o teor de umidade de equilíbrio foi obtido após aproximadamente 580 minutos de secagem na temperatura de $60{ }^{\circ} \mathrm{C}$.

A Figura 05 apresenta a curva de secagem das sementes de abóbora para a temperatura de $70^{\circ} \mathrm{C}$.

Figura 05: Curva de secagem das sementes de abóbora obtida pelo modelo matemático de Midilli na temperatura de $70^{\circ} \mathrm{C}$

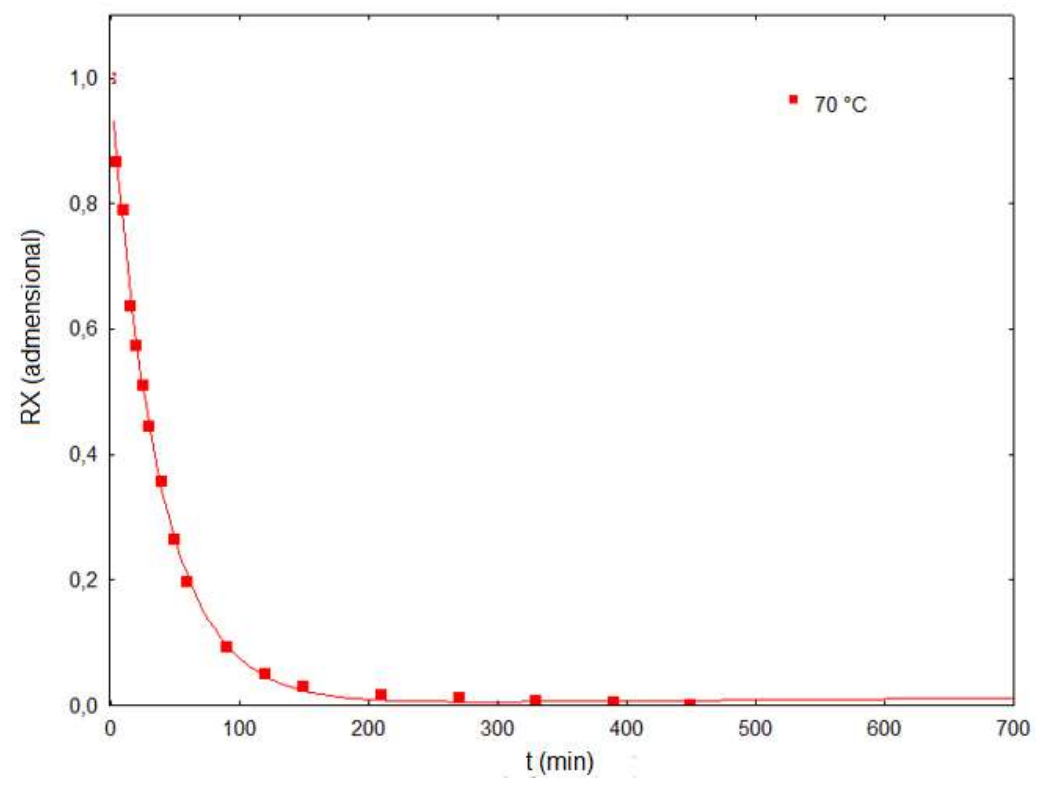

Fonte: Própria (2021)

Ao observar a Figura 05, pode-se verificar que o teor de umidade de equilíbrio foi obtido após aproximadamente 450 minutos de secagem na temperatura de $70{ }^{\circ} \mathrm{C}$. 
Através das Figuras 03, 04 e 05, pode-se observar o incremento da temperatura do ar de secagem favorece a transferência de massa do produto, consequentemente diminui o teor de umidade de equilíbrio dinâmico e o tempo de secagem.

A Figura 06 apresenta as curvas de secagem de sementes de abóbora obtidas pelo modelo de Midilli nas temperaturas de $50, \quad 60$ e $70 \quad{ }^{\circ} \mathrm{C}$.

Figura 06: Curvas de secagem das sementes de abóbora obtida pelo modelo matemático de Midilli nas temperaturas de 50,60 e $70{ }^{\circ} \mathrm{C}$

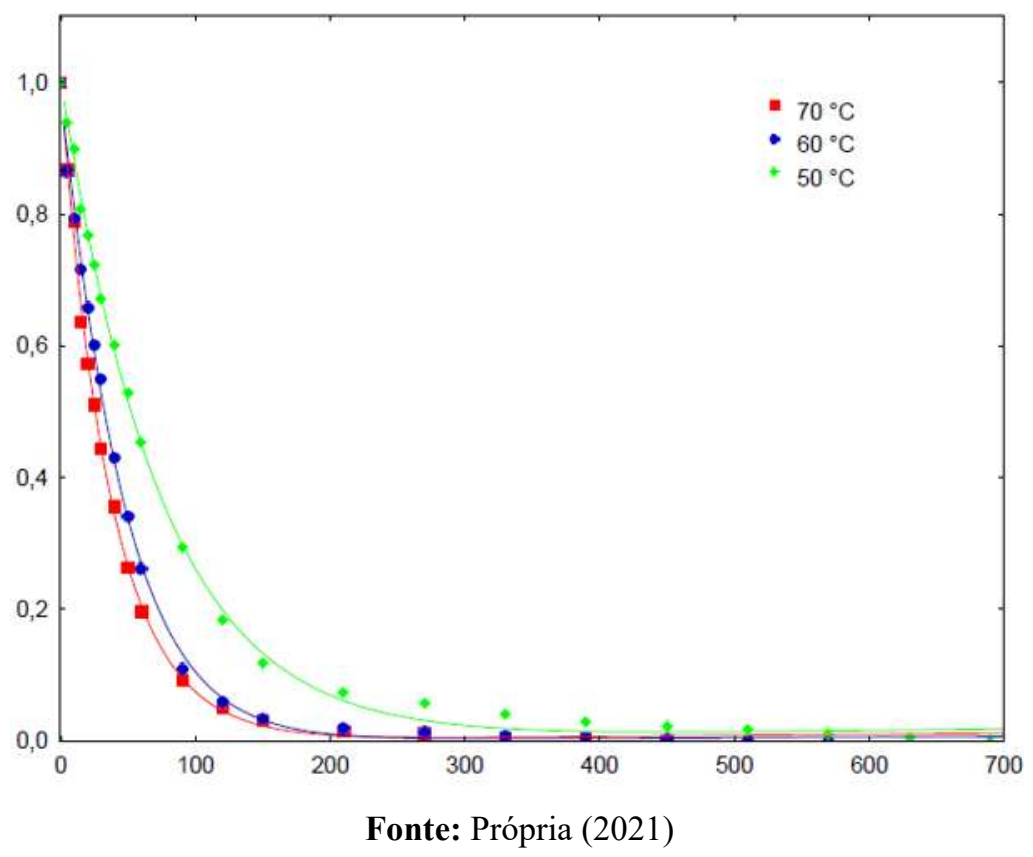

Os tempos de secagem variaram de 450 a 600 minutos, sendo o menor tempo de secagem obtido na maior temperatura. De acordo com Ribeiro et al. (2019), o acréscimo da temperatura do ar de secagem proporciona maior gradiente de umidade entre o produto e o ar, ocasionando assim maior taxa de remoção de água do produto.

Segundo Corrêa et al. (2017), este fenômeno ocorre porque em temperaturas mais elevadas as amostras apresentam maior agitação de suas moléculas (maior mobilidade das moléculas de água dentro dos alimentos), reduzindo assim a resistência interna ao transporte de massa. Já a resistência externa ao transporte de massa diminui devido ao aumento do gradiente de pressão da água entre as fases, líquida e vapor. Além disso, altas temperaturas de secagem fornecem a energia requerida para ultrapassar o calor latente da mudança de fase, que ocorre durante a evaporação da água.

A Figura 07 apresenta o gráfico obtido para as taxas de secagem das sementes de 
abóbora variando as temperaturas do ar de secagem $\left(50,60\right.$ e $\left.70^{\circ} \mathrm{C}\right)$, a partir da derivada do modelo de Page.

Figura 07: Gráfico das taxas de secagem de sementes de abóbora, obtidas pela derivada do modelo de Page (temperaturas de 50,60 e $70^{\circ} \mathrm{C}$ )

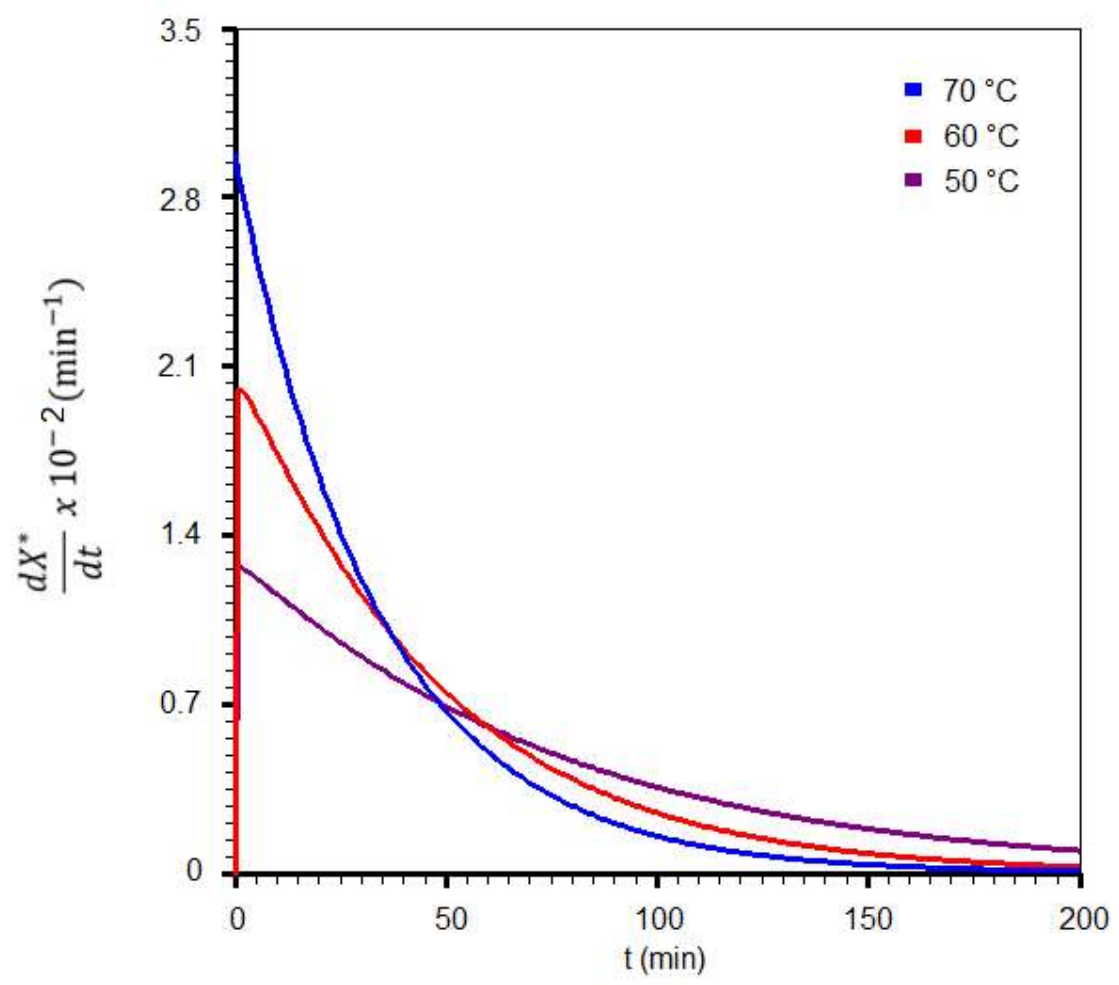

Fonte: Própria (2021)

A Figura 07 apresenta as curvas que representam as taxas de secagem em todas as temperaturas aplicadas $\left(50,60\right.$ e $\left.70^{\circ} \mathrm{C}\right)$. A partir da avaliação dos gráficos, pode-se inferir que o produto apresenta taxas de secagem crescentes nos instantes iniciais do processo, este período é denominado por período de indução. Ao finalizar o período de indução, obtêm-se a maior taxa de secagem no processo, que variou de $1,3 \times 10^{-2} \mathrm{~min}^{-1}$ (temperatura de $50^{\circ} \mathrm{C}$ ) a $3,0 \times 10^{-2} \mathrm{~min}^{-1}$ (temperatura de $70^{\circ} \mathrm{C}$ ), constatou-se a taxa de secagem é proporcional ao gradiente de umidade no produto. Após alcançar a maior taxa de secagem possível, inicia-se um período de taxa decrescente que ocorre por causa da redução do gradiente de umidade no produto. Pois, ao longo do processo, ocorre a diminuição do teor de água do produto e diminuição dos gradientes apresentados, consequentemente percebe-se a diminuição da taxa de secagem (-dX * / dt) dos instantes iniciais até o final do processo, quando a taxa de secagem tem um valor nulo, indicando que uma condição de equilíbrio foi alcançada. Santos et al. (2019b), em seus estudos 
sobre a secagem de fatias de pêssego, também encontraram maiores valores de taxa de secagem durante os instantes iniciais do processo, nos quais o produto apresentou maiores valores da razão de umidade.

Feitosa et al. (2017), em seus estudos de secagem de polpa de murta também observaram que as maiores taxas de secagem foram observadas nos instantes iniciais do processo. Este fato pode ser explicado porque nos instantes iniciais do processo há um maior gradiente entre a umidade do material e a umidade do ar, proporcionando assim maiores taxas de transferência de massa.

\section{CONCLUSÕES}

Através do presente estudo, foi possível constatar que o processo de secagem é uma ótima alternativa para promover o aproveitamento das sementes de abóbora. Observou-se que o parâmetro "a" e o " $\mathrm{k}$ " são diretamente proporcionais ao aumento da temperatura do ar de secagem, comportamento contrário foi observado com relação ao parâmetro "b" que diminuiu com o acréscimo da temperatura do ar de secagem. O parâmetro "n", diferente dos demais, não apresentou correlação com a temperatura do ar de secagem.

Todos os modelos matemáticos apresentaram ajustes satisfatórios aos dados experimentais obtidos em todas as temperaturas aplicadas, porém o modelo de Midilli apresentou o melhor ajuste aos dados experimentais obtidos, pois exibiu valores superiores de $\mathrm{R}^{2}$ e valores inferiores de DQM, quando comparado aos demais. Foi verificada uma redução do tempo de secagem com o acréscimo da temperatura do ar de secagem, pois a maior taxa de secagem foi observada ao aplicar a maior temperatura $\left(70^{\circ} \mathrm{C}\right)$.

\section{REFERÊNCIAS}

ALVES, Jordana dos Santos. Modelagem matemática de secagem da casca de abóbora moranga (Cucurbita maxima.). 2019. 29 f. Trabalho de conclusão de curso (Graduação em Engenharia de Alimentos), IFECT, 2019.

ALVES, R. A.; QUEIROZ, A. J. M.; FIGUEIRÊDO, R. M. F. DE; SILVA, W. P.; GOMES, J. P. Solar drying of cowpea bean combined with drying in a heat accumulator dryer. Revista Brasileira de Engenharia Agrícola e Ambiental, v.23, n.9, p.709-715, 2019.

AMARAL, L. F.; FERREIRA, I. M.; SANTOS, L. V. N.; SILVA, A. M. O.; FAGUNDES, A. A.; CARVALHO, M. G. Biscuit with spices and corn flours and pumpkin seed: development and quality assessment. Science and Food Technology, v.14, e33380, p. 1-17, 2019.

BENSEDDIK, A.; AZZI, A.; ZIDOUNE, M. N.; \& ALLAF, K. Mathematical empirical models of thin-layer airflow drying kinetics of pumpkin slice. Engineering in Agriculture, 
Environment and Food, v.1, p.1-12, 2018. doi:10.1016/j.eaef.2018.07.003

CORRÊA, J. L. G.; RASIA, M. C.; MULET, A.; CÁRCEL, J. A. Influence of ultrasound application on both the osmotic pretreatment and subsequent convective drying of pineapple (Ananas comosus). Innovative Food Science \& Emerging Technologies, v.41, p.284-291, 2017.

FEITOSA, R. M.; FIGUEIRÊDO, R. M. F.; QUEIROZ, A. J. M.; LIMA, F. C. S.; OLIVEIRA, E. N. A. Drying and characterization of myrtle pulp. Revista Brasileira de Engenharia Agrícola e Ambiental, v.21, n.12, p.858-864, 2017.

GONELI, A. L. D.; CORRÊA, P. C.; MAGALHÃES, F. E. A.; BAPTESTINI, F. M. Contração volumétrica e forma dos frutos de mamona durante a secagem. Acta Scientiarum, v.33, n.1, p.1-8, 2011.

HENDERSON, S. M., \& PABIS, S. Grain drying theory I: Temperature effect on drying coefficient. Journal of Agricultural Engineering Research, v.6, p.169-174, 1961.

HERNÁNDEZ-SANTOS, Betsabé; RODRÍGUEZ-MIRANDA, Jesús; HERMAN-LARA, Erasmo; TORRUCO-UCO, Juan G.; CARMONA-GARCÍA, Roselis; JUÁREZBARRIENTOS, José M.; CHÁVEZ-ZAMUDIO, Rubí; MARTÍNEZ-SÁNCHEZ, Cecilia E.. Effect of oil extraction assisted by ultrasound on the physicochemical properties and fatty acid profile of pumpkin seed oil (Cucurbita pepo). Ultrasonics Sonochemistry, [S.L.], v. 31, p. 429436, jul. 2016.

MALDONADE, Iriani Rodrigues; LOZADA, Maria Isabel Ordoñez; AMARO, Geovani; OLIVEIRA, Livia de Lacerda de; LUENGO, Rita de Fátima; MACHADO, Eleuza Rodrigues. Propriedades funcionais e nutracêuticas de sementes de cucurbitáceas. Brasília, DF: Embrapa Hortaliças, 2019. 22 p.: il. color. (Boletim de pesquisa e desenvolvimento / Embrapa Hortaliças, ISSN 1677-2229; 197).

MARTINS, E. A.; LAGE, E. Z.; GONELI, A. L. D.; HARTMANN FILHO, C. P.; LOPES, J. G. Drying kinetics of Serjania marginata Casar leaves. Revista Brasileira de Engenharia Agrícola e Ambiental, v.19, n.3, p.238-244, 2015.

MIDILLI, A.; KUCUK, H.; YAPAR, Z.; A new model for single-layer drying. Drying Technology. v.20, p.1503-1513, 2002.

MOREIRA, I. S.; SILVA, W. P.; CASTRO, D. S.; SILVA, L. M. M.; GOMES, J. P.; SILVA, C. M. D. P. S. Production of kiwi snack slice with different thickness: Drying kinetics, sensory and physicochemical analysis. v.12, p.778-787, 2018.

MOSCON, E. S.; MARTIN, S.; SPEHAR, C. R.; DEVILLA, I. A.; RODOLFO JÚNIOR, F. Drying kinetic of quinoa grain (Chenopodium quinoa $W$.). Revista Engenharia na Agricultura, v.25, n.4, p.318-328, 2017.

PAGE, G. E. Factors influencing the maximum rates of air drying shelled corn in thin layers. 44 p. MSc. Thesis. West Lafayette, Purdue University, 1949.

RESENDE, G. M; BORGES, R. M. E; GONÇALVES, N. P. S. Produtividade da cultura da abóbora em diferentes densidades de plantio no Vale do São Francisco. Horticultura 
Brasileira, v. 31, p. 504-508, 2013.

RIBEIRO, V. H. A.; ALMEIDA, R. L. J.; SANTOS, N. C.; BARROS, S. L.; NASCIMENTO, A. P. S. Mathematical modeling of apple drying kinetics. Revista Higiene Alimentar, v.33, n.288/289, p.3474-3478, 2019.

SANTOS, N. C.; SILVA, V. M. A.; RIBEIRO, V. H. A.; BARROS, S. L.; NASCIMENTO, A. P. S.; ARAÚJO, A. J. B.; ALMEIDA, R. L J. Modelagem matemática da cinética de secagem de nozes (juglans regia 1.) sem casca. In: Francisco et al. (Org.). Caderno de Ciência Pesquisa e Inovação. EPGRAF, Campina Grande-PB, v.2, n.2, p.71-77, 2019.

SANTOS, N. C., SILVA, W. P., BARROS, S. L., ALMEIDA, R. L. J., DE BRITO ARAÚJO, A. J., \& DA SILVA NASCIMENTO, A. P. Red rice (Oryza sativa L .) use in flour production: Convective drying and bioactive quality. Journal of Food Process Engineering, v.1, p.1-10, 2020 .

SILVA, Beatriz Santos da. Estudo cinético de secagem de abóbora (Cucurbita moschata) in natura e submetida ao congelamento. 2016. $57 \mathrm{f}$. Trabalho de conclusão de curso (Engenharia de Alimentos), UFPB, 2016.

SILVA, E. C. O.; SILVA, W. P.; GOMES, J. P.; SILVA, C. M. S. P. S.; ALEXANDRE, H. V.; FARIAS, V. S. O.; MELO, B. A.; QUEIROJ, A. J. M.; FIGUEREDO, R. M. F. Drying of albedo and whole peel of yellow passion fruit. Journal of Agricultural Science, v.11, n.6, p.501-509, 2019.

SILVA, Gabriella Dias da. Influência do uso do vácuo e/ou ultrassom como prétratamento em parâmetros de qualidade do melão (Cucumis melo var. cantalupensis naud.) seco. 2016. 106 f. Dissertação ( Programa de Pós-Graduação em Nutrição), UFPE, 2016.

SILVA, Virgínia Mirtes de Alcântara; SANTOS, Newton Carlos; BARROS, Sâmela Leal; ALMEIDA, Raphael Lucas Jacinto; RIBEIRO, Victor Herbert de Alcântara; MELO, Mylena Olga Pessoa. Conservação de sementes de abóbora através de secagem convectiva. Caderno Verde de Agroecologia e Desenvolvimento Sustentável, [S.L.], v. 9, n. 7, p. 7098, 8 jan. 2020.

VALE, Camilla Pereira do; LOQUETE, Fernanda Cristina Cavalcante; ZAGO, Morgana Gris; CHIELLA, Pietra Vilani; BERNARDI, Daniela Miotto. Composição e propriedades da semente de abóbora. Fag Journal Of Health (Fjh), [S.L.], v. 1, n. 4, p. 79-90, 20 dez. 2019.

VIEIRA, Danise Medeiros; BARROS, Sâmela Leal; SILVA, Vírgínia Mirtes de Alcântara; SANTOS, Newton Carlos; NASCIMENTO, Amanda Priscila Silva; MELO, Mylena Olga Pessoa. Cinética de secagem e sua influência nas dimensões de sementes de abóbora sem casca. Revista Verde de Agroecologia e Desenvolvimento Sustentável, [S.L.], v. 14, n. 5, p. 665670, 20 dez. 2019. 